\title{
Suppression of background emission for efficient single-photon generation in micropillar cavities
}

Wang, Bi-Ying; Häyrynen, Teppo; Vannucci, Luca; Jacobsen, Martin Arentoft; Lu, Chao-Yang; Gregersen, Niels

Published in:

Applied Physics Letters

Link to article, DOI:

$10.1063 / 5.0044018$

Publication date:

2021

Document Version

Peer reviewed version

Link back to DTU Orbit

Citation (APA):

Wang, B-Y., Häyrynen, T., Vannucci, L., Jacobsen, M. A., Lu, C-Y., \& Gregersen, N. (2021). Suppression of background emission for efficient single-photon generation in micropillar cavities. Applied Physics Letters, 118(11), [114003]. https://doi.org/10.1063/5.0044018

\section{General rights}

Copyright and moral rights for the publications made accessible in the public portal are retained by the authors and/or other copyright owners and it is a condition of accessing publications that users recognise and abide by the legal requirements associated with these rights.

- Users may download and print one copy of any publication from the public portal for the purpose of private study or research.

- You may not further distribute the material or use it for any profit-making activity or commercial gain

- You may freely distribute the URL identifying the publication in the public portal 


\section{Suppression of background emission for efficient single-photon generation in micropillar cavities}

Bi-Ying Wang, ${ }^{1,2}$ Teppo Häyrynen, ${ }^{2}$ Luca Vannucci, ${ }^{2}$ Martin Arentoft Jacobsen, ${ }^{2}$ Chao-Yang Lu, ${ }^{1}$ and Niels Gregersen ${ }^{2,}$ a)

${ }^{1)}$ Hefei National Laboratory for Physical Sciences at Microscale, University of Science and Technology of China, Hefei, Anhui, 230026, China

${ }^{2)}$ DTU Fotonik, Department of Photonics Engineering, Technical University of Denmark, Ørsteds Plads, Building 343, DK-2800 Kongens Lyngby, Denmark

(Dated: 24 February 2021)

We optimize the efficiency of a quantum-dot-based micropillar single-photon source by minimizing the spontaneous emission into unwanted background modes. We perform a numerical investigation of the background emission, where we identify a semi-periodic enhancement with diameter. At these peaks of the background emission, the efficiency is reduced by $\sim 20 \%$, and we show how this reduction can be avoided simply by choosing a diameter away from the peaks. Finally, we analyze the discrepancy between the Purcell factor estimated from experimental lifetime measurements and the true Purcell factor.

Within the fields of optical quantum computation ${ }^{1}$ and communication, ${ }^{2}$ a key component is the single-photon source $^{3,4}$ (SPS) capable of emitting single indistinguishable photons on demand. A main figure of merit is the efficiency $\varepsilon$ defined as number of collected photons in the detection optics per trigger. Since the success probability of a multi-photon interference experiment with $N$ photons scales as $\varepsilon^{N}$, the realization of scalable optical quantum information technology requires increasing the efficiency towards unity. The semiconductor quantum dot (QD) embedded in a semiconductor host material has recently emerged as a promising platform for highly efficient deterministic generation of single photons. While the collection efficiency for a QD in a bulk material is $\sim 0.02$ due to the reflection at the semiconductor-air interface, the light emission can be improved by placing the QD in an optical microcavity. The most succesful SPS microcavity designs today include the narrowband vertical micropillar cavity, ${ }^{5-7}$ the broad-band planar "bullseye" cavity ${ }^{5,8,9}$ and the tunable open cavity, ${ }^{10}$ where the emission rate into the cavity mode is enhanced by the Purcell factor $F_{\mathrm{p}}=\Gamma_{\mathrm{c}} / \Gamma_{\text {bulk }}$, where $\Gamma_{\mathrm{c}}$ and $\Gamma_{\text {bulk }}$ are the emission rates into the cavity mode and in a bulk medium respectively. The efficiency is then proportional to the spontaneous emission $\beta$ factor ${ }^{4,11}$ given by $\beta=\frac{F_{\mathrm{p}}}{F_{\mathrm{p}}+\Gamma_{\mathrm{b}} / \Gamma_{\text {bulk }}}$, where $\Gamma_{\mathrm{b}}$ is the emission rate into background modes.

As alternative to simply increasing $F_{\mathrm{p}}$, a less explored strategy for realizing near-unity efficiency relies on the suppression of the emission $\Gamma_{\mathrm{b}}$ into background modes. In the photonic nanowire SPS ${ }^{12}$ the suppression results simply from a screening effect ${ }^{13}$ occurring in the singlemode diameter regime. In the large-diameter regime, the introductions of a gold coating ${ }^{14}$ and a radial distributedBragg reflector ${ }^{15}$ have been proposed to control the background emission. However, a detailed analysis of suppression mechanisms in the large-diameter regime has not

a) Author to whom correspondence should be addressed; Electronic mail: ngre@fotonik.dtu.dk yet been performed. In a recent work, ${ }^{16}$ the micropillar SPS performance was investigated, and here periodic decreases in $\beta$ as function of diameter were observed, attributed simply to variations in the background emission rate. However, the origin of these variations was not investigated, and an understanding of the positions of the peaks and valleys of the background emission rate with respect to diameter is needed to maximize the efficiency during the design process.

In this Letter, we employ the Fourier Modal Method $^{17-19}$ (FMM) to access the background emission rate, first in a bare nanowire geometry and subsequently in a full micropillar cavity. We observe periodic variations in the background rate with one-to-one correspondence between the peaks in the background emission and the onset of new guided modes as function of pillar diameter. We use this observation to present a guideline for achieving optimal micropillar SPS performance. Finally, we exploit our access to the background emission to assess the validity of a commonly used experimental procedure to estimate the Purcell factor.

In the FMM, the structure under study is divided into uniform layers along the propagation axis $z$. The electric

(a) Infinite nanowire (b) Micropillar

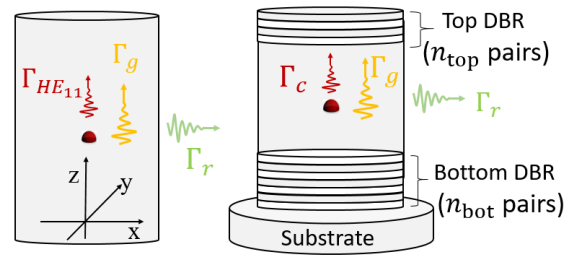

FIG. 1. Schematic of the infinite GaAs nanowire (a) and the micropillar (b), where the QD is represented by a red dot. The micropillar features distributed Bragg reflectors (DBRs) with $n_{\text {top }}\left(n_{\text {bot }}\right)$ layer pairs above (below) the QD. 
field in each layer is expanded on eigenmodes $\mathbf{E}_{ \pm, j}(\mathbf{r})=$ $\mathbf{e}_{j}\left(\mathbf{r}_{\perp}\right) e^{ \pm i \beta_{j} z}$, which are connected at each side of a layer interface using the $\mathrm{S}$ matrix formalism. ${ }^{19}$ We model the $\mathrm{QD}$ as a classical point dipole $\mathbf{p}$ with in-plane orientation and harmonic time dependence at the frequency $\omega$. The current density is then given by $\mathbf{J}(\mathbf{r})=-i \omega \mathbf{p} \delta\left(\mathbf{r}-\mathbf{r}_{\mathrm{d}}\right)$ for a QD at the position $\mathbf{r}_{\mathrm{d}}$. In the case of the infinite nanowire illustrated in Fig. 1(a), the emitted power $P_{j}$ into the $j$ th mode is simply given by ${ }^{19,20}$

$$
P_{j}=-\frac{1}{2} \int_{V} \operatorname{Re}\left[\mathbf{J}^{*}(\mathbf{r}) \cdot \mathbf{E}_{j}(\mathbf{r})\right] d V=\frac{\omega}{2} \operatorname{Im}\left[\mathbf{p} \cdot \mathbf{E}_{j}\left(\mathbf{r}_{\mathrm{d}}\right)\right] .
$$

The spontaneous emission rate $\Gamma_{j}$ into the $j$ th mode is then determined from (1) $\mathrm{as}^{20}$

$$
\frac{\Gamma_{j}}{\Gamma_{\text {bulk }}}=\frac{P_{j}}{P_{\text {bulk }}},
$$

where $P_{\text {bulk }}$ is the power emitted in a homogeneous medium. For the micropillar cavity shown in Fig. 1(b), the fundamental resonant cavity mode $\mathbf{E}_{\mathrm{c}}(\mathbf{r})$ is found by diagonalization of a roundtrip matrix as discussed in Ref. 16 , and the emission rate $\Gamma_{\mathrm{c}}$ into the cavity is again obtained from Eqs. $(1,2)$ using the replacement $j \rightarrow c$. State-of-the-art nano-positioning techniques ${ }^{5,21,22}$ allow for excellent spatial QD position control with accuracy below $10 \mathrm{~nm}$, and we thus assume a QD placed exactly on-axis. Finally, the refractive indices used in the simulations are determined using the model in Ref. 23 at 4 $\mathrm{K}$.

For the infinite GaAs nanowire depicted in Fig. 1(a) with refractive index $n_{1}\left(n_{2}\right)$ of the core (cladding) material, each mode $j$ can be categorized in terms of its propagation constant $\beta_{j}$ and the wave number $k_{0}=\omega / c$ in vacuum: Modes satisfying $n_{2} k_{0}<\beta_{j} \leq n_{1} k_{0}$ are guided modes, whereas those fulfilling $\beta_{j}<n_{2} k_{0}$ are radiation modes. In the following, we denote the emission rate into the fundamental waveguide mode as $\Gamma_{\mathrm{HE}_{11}}$, the rate into higher-order (non- $\mathrm{HE}_{11}$ ) guided modes as $\Gamma_{\mathrm{g}}$, and finally $\Gamma_{\mathrm{r}}$ is the rate into radiation modes. The total background emission rate then reads $\Gamma_{\mathrm{b}}=\Gamma_{\mathrm{g}}+\Gamma_{\mathrm{r}}$, while the total emission rate is $\Gamma_{\mathrm{t}}=\Gamma_{\mathrm{HE}_{11}}+\Gamma_{\mathrm{b}}$.

The calculated emission rates $\Gamma_{\mathrm{b}}, \Gamma_{\mathrm{g}}$ and $\Gamma_{\mathrm{r}}$ as function of diameter for the infinite nanowire are presented in Fig. 2(a), and emission rates $\Gamma_{j}$ into selected individual modes are shown in Fig. 2(b). For small diameters $d / \lambda<0.3$, the background emission $\Gamma_{\mathrm{b}}$ is suppressed due to the screening effect ${ }^{13}$ and light emission is predominantly into the fundamental $\mathrm{HE}_{11}$ mode. ${ }^{4,12,13}$ For larger diameters, $\Gamma_{\mathrm{b}}$ displays a periodic oscillatory behavior around the bulk value, which can be understood from inspection of the emission rates $\Gamma_{j}$ in Fig. 2(b) into individual guided modes. For an on-axis dipole emitter with in-plane orientation, light is emitted exclusively into the two classes of $\mathrm{HE}_{1, m+1}$ and $\mathrm{EH}_{1, m}$ modes with identical mode cutoff diameters $d_{m}$ given by ${ }^{24}$

$$
\frac{d_{m}}{\lambda}=\frac{z_{1, m}}{\pi \sqrt{n_{1}^{2}-n_{2}^{2}}}, \quad m=1,2,3 \ldots
$$

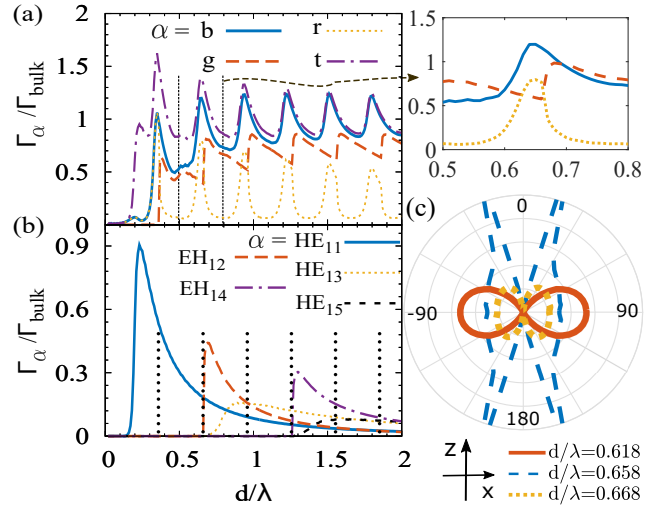

FIG. 2. Infinite nanowire geometry: (a) Total $\left(\Gamma_{t}\right)$, background $\left(\Gamma_{\mathrm{b}}\right)$, guided higher-order modes $\left(\Gamma_{\mathrm{g}}\right)$ and radiation $\left(\Gamma_{\mathrm{r}}\right)$ emission rates as a function of diameter $d / \lambda$. A zoom of the region $d / \lambda \in[0.5,0.8]$ is shown on the right. (b) Emission rates into individual guided modes with vertical dotted lines marking the mode cutoffs $d_{m} / \lambda$. (c) Far field emission pattern of the radiation modes in the $x-z$ plane for a nanowire aligned along the $z$ axis for the diameters $d / \lambda=0.618$ (blue), $d / \lambda=0.658$ (red) and $d / \lambda=0.668$ (yellow). $n_{\mathrm{GaAs}}=$ $3.5015 .^{23}$

where $z_{1, m}$ is the $m$ th zero point of the Bessel $J_{1}(x)$ function ( $\mathrm{HE}_{11}$ has no cutoff value). The cutoffs are indicated in Fig. 2(b) using vertical dotted lines, and we observe that the emission into an $\mathrm{EH}_{1, m}$ mode peaks abruptly shortly after the cutoff followed by a uniform decrease towards zero due to the increasing mode area, whereas the $\mathrm{HE}_{1, m+1}$ emission features a slower initial increase with a peak near the subsequent cutoff. This behavior is reflected in the total emission rate $\Gamma_{\mathrm{g}}$ into higher-order guided modes in Fig. 2(a), where a peak due to the onset of a new $\mathrm{EH}_{1, m}$ mode appears just after the $d_{m}$ cutoff. Due to the lower amplitude of the $\mathrm{HE}_{1, m+1}$ emission, a corresponding peak from the $\mathrm{HE}_{1, m+1}$ mode is not directly observed. The emission into radiation modes follows a similar oscillatory trend, where the peak in $\Gamma_{r}$ occurs just before a cutoff value. To understand this variation in $\Gamma_{\mathrm{r}}$, we present the far field emission pattern for the radiation modes in the $x-z$ plane at diameters near the $m=2$ cutoff $\left(d_{2} / \lambda \approx 0.666\right)$ in Fig. $2(\mathrm{c})$. For $d / \lambda=0.618$, the power is emitted predominantly in the lateral direction, however as the diameter increases to $d / \lambda=0.658$, the power is strongly enhanced and directed towards the vertical direction $z$. Finally, for a diameter just above the cutoff $(d / \lambda=0.668)$, the emission is reduced and again directed in the lateral direction.

We may thus describe the variation in total background emission rate $\Gamma_{\mathrm{b}}=\Gamma_{\mathrm{g}}+\Gamma_{\mathrm{r}}$ in terms of enhanced emission into a semi-guided mode which peaks just before the onset $d_{m}$ of a new guided mode. When the di- 


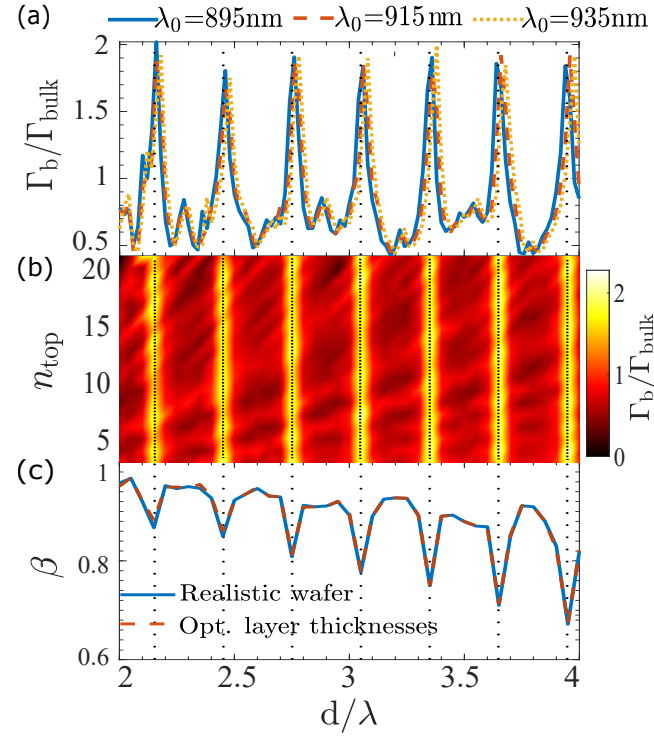

FIG. 3. Micropillar geometry: (a) Normalized background emission rates for three design wavelengths as function of diameter. (b) Background emission as function of top DBR layer pairs $n_{\text {top }}$ and of diameter, encoded in a false color scale, with $\lambda_{0}=895 \mathrm{~nm}$ and $n_{\text {bot }}=2 n_{\text {top }}$. (c) Spontaneous emission $\beta$ factor as function of diameter for layer thicknesses optimized for $\lambda_{0}=895 \mathrm{~nm}$ for all diameters (red) and for a realistic fixed-layer-thickness wafer chosen such that $\lambda_{0}=895$ $\mathrm{nm}$ for $d_{0}=3 \mu \mathrm{m}$ (blue). We set $n_{\text {bot }} / 2=n_{\text {top }}=15$ in (a) and (c). Vertical dotted lines mark the mode cutoffs $d_{m} / \lambda$ We use respectively $n_{\mathrm{GaAs}}=3.5015,3.4859$ and 3.4721 and

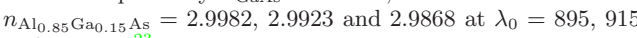
and $935 \mathrm{~nm}^{23}$

ameter increases past the cutoff, light emission into the semi-guided mode is transformed to emission into two fully guided $\mathrm{HE}_{1, m+1}$ and $\mathrm{EH}_{1, m}$ modes. After the cutoff point, the emission is then predominantly into guided modes until the next onset $d_{m+1}$ of the next guided modes, where the cycle repeats itself.

We now investigate the micropillar geometry illustrated in Fig. 1(b) consisting of a vertical $\lambda$ cavity with a $\mathrm{QD}$ at the center sandwiched between two distributed Bragg reflectors (DBRs) featuring $n_{\text {top }}\left(n_{\text {bot }}\right)$ pairs of GaAs $/ \mathrm{Al}_{0.85} \mathrm{Ga}_{0.15} \mathrm{As}$ in the top (bottom) DBR. We consider an asymmetric structure always satisfying $n_{\text {bot }}=$ $2 n_{\text {top }}$ to avoid leakage of light into the substrate. Unless otherwise stated, diameter-dependent cavity and DBR layer thicknesses are chosen to place the micropillar resonance at a design wavelength $\lambda_{0}=895 \mathrm{~nm}$ for all diameters as discussed in Ref. 16. The total emission rate for the micropillar is then given by $\Gamma_{\mathrm{t}}=\Gamma_{\mathrm{c}}+\Gamma_{\mathrm{b}}=$ $F_{\mathrm{p}} \Gamma_{\text {bulk }}+\Gamma_{\mathrm{b}}$
The calculated background emission rate $\Gamma_{\mathrm{b}}$ for the micropillar cavity for three design wavelengths is presented in Fig. 3(a), and a periodic oscillatory behavior in the $\Gamma_{\mathrm{b}} / \Gamma_{\text {bulk }} \in[0.6,2]$ interval is observed. Similarly to the nanowire case, we notice that the peaks in the background emission appear exactly at the position of the $\mathrm{HE}_{1, m+1}$ and $\mathrm{EH}_{1, m}$ mode cutoffs (vertical dotted lines) for all design wavelengths. To explain this behavior, we note that the physics of the onset of new waveguide modes in the GaAs cavity is identical to that of the infinite nanowire, and the peaks may thus again be explained by enhanced emission into a semi-guided mode initially not subject to cavity effects. As the semi-guided mode is subsequently transformed to a guided higher-order cavity mode, anti-resonant cavity effects lead to destructive interference and a corresponding reduction of the emission into the higher-order cavity mode. This explains why, unlike the infinite nanowire where the background emission oscillates increasingly closer to the bulk value for increasing diameter, destructive interference effects in the cavity allow for $\Gamma_{\mathrm{b}} / \Gamma_{\text {bulk }}$ valleys of $\sim 0.6$ in the entire diameter interval considered. In these valleys, additional minor peaks are observed resulting from the complex interplay between resonant and anti-resonant cavity effects.

The influence of the number of layer pairs on the background emission is investigated in Fig. 3(b). We observe a weak modulation for an increasing value of $n_{\text {top }}$ due to coupling to higher-order Bloch modes in the DBRs propagating outside the stopband, a mechanism also responsible for oscillations of the cavity $Q$ factor ${ }^{25,26}$ with diameter. However, importantly, we observe that the overall positions of the peaks and valleys are independent of the number of layer pairs. Additionally, we present the spontaneous emission $\beta$ factor in Fig. 3(c) computed for two different structures: (i) An optimized structure, where the layer thicknesses are varied with diameter to keep the resonant wavelength $\lambda_{0}=895 \mathrm{~nm}$ for all diameters. (ii) A realistic wafer structure with fixed layer thicknesses designed to have a resonance wavelength $\lambda_{0}=895 \mathrm{~nm}$ at a design diameter $d_{0}=3 \mu \mathrm{m}$. In the second structure, the cavity resonance differs from $\lambda_{0}$ for $d \neq d_{0}$. However, we observe good agreement between the two cases, which we attribute to the limited variation $(\sim 5 \mathrm{~nm})$ of the resonance wavelength in the diameter range considered. For this $15 / 30$ DBR configuration, we also note that the factor of $\sim 3$ variation in $\Gamma_{\mathrm{b}}$ observed in Fig. 3(a) leads to a sizeable decrease up to $\sim 20 \%$ in the $\beta$ factor.

In summary, we observe that the peaks in the back-

TABLE I. Optimal $d_{m}^{\text {opt }}$ values (in units of $\mathrm{nm}$ ) for different design wavelengths $\lambda_{0}$ with optimized layer thicknesses chosen for each diameter to match the desired $\lambda_{0}$.

\begin{tabular}{rccccccc}
\hline \hline$\lambda_{0}$ & $d_{7}^{\text {opt }}$ & $d_{8}^{\text {opt }}$ & $d_{9}^{\text {opt }}$ & $d_{10}^{\text {opt }}$ & $d_{11}^{\text {opt }}$ & $d_{12}^{\text {opt }}$ & $d_{13}^{\text {opt }}$ \\
\hline 895 & 2066 & 2333 & 2600 & 2866 & 3133 & 3400 & 3666 \\
915 & 2122 & 2396 & 2670 & 2945 & 3219 & 3493 & 3767 \\
935 & 2178 & 2460 & 2741 & 3022 & 3303 & 3585 & 3866 \\
\hline \hline
\end{tabular}


ground emission appear at scaled diameters given by the scaled guided mode cutoff $d_{m} / \lambda$ values independently of the number of layer pairs. While the resonance wavelength defines the $d_{m} / \lambda$ scale, it does not otherwise change the peak positions. Since the separation between successive zero points $z_{1, m}$ and $z_{1, m+1}$ of the Bessel function $J_{1}(x)$ for large values of $m$ is well approximated by $\Delta z=\pi$. the cutoff separation can be written as $\Delta d \approx \lambda / \sqrt{n_{1}^{2}-n_{2}^{2}}$. The optimal design recipe for efficient single-photon emission from micropillars thus involves a selection from the sequence $d_{m}^{\text {opt }}$ of optimal discrete diameters

$$
d_{m}^{\mathrm{opt}}=d_{m}+\frac{\Delta d}{2}=\frac{\lambda\left(2 z_{1, m}+\pi\right)}{2 \pi \sqrt{n_{1}^{2}-n_{2}^{2}}},
$$

with example values given in Table I. For these diameters, the normalized background emission in Fig. 3(a) takes an average value of $\sim 0.6$ leading to an estimated spontaneous emission $\beta$ factor of $\beta \approx F_{\mathrm{p}} /\left(F_{\mathrm{p}}+0.6\right)$. In this work, a perfect structure without fabrication imperfection is considered. While sidewall roughness leads to a reduction in extraction efficiency, it does not alter the positions of peaks and dips in $\beta$ as function of diameter (cf. Fig. 5 in Ref. 16), and the optimal sequence in Eq. (4) remains valid even in the presence of sidewall roughness.

We note that, even at the shortest design wavelength $\lambda_{0}=895 \mathrm{~nm}$, we obtain a separation $\Delta d \approx 267 \mathrm{~nm}$ large enough to avoid the cutoff values $d_{m}$ during the etching process. Moreover, an uncertainty in resonance wavelength of $\delta \lambda / \lambda \sim 1 \%$ due to imperfections in the device fabrication will result in a similar uncertainty $\delta d_{m} / d_{m} \sim 1 \%$ from Eq. (3). However, this is sufficiently small compared to the separation $\Delta d / d \sim 5 \%$ between emission peaks and valleys in the $d \in[2,4] \mu \mathrm{m}$ interval such that the background emission peaks can be avoided even in the presence of realistic device fabrication imperfection.

Finally, we investigate the validity of a frequently used procedure ${ }^{22,27}$ to experimentally estimate the Purcell factor. Here, time-resolved photoluminescence lifetime measurements are used to measure the (total) spontaneous emission rate first on resonance and subsequently offresonance e.g. using temperature tuning of the QD emission line. For the resonant (r) configuration, the normalized total emission rate is

$$
\frac{\Gamma_{\mathrm{t}}^{\mathrm{r}}}{\Gamma_{\text {bulk }}}=F_{\mathrm{p}}+\frac{\Gamma_{\mathrm{b}}^{\mathrm{r}}}{\Gamma_{\mathrm{bulk}}}
$$

whereas for the non-resonant (nr) configuration, where the QD detuning is much larger than the cavity bandwidth, we simply have

$$
\frac{\Gamma_{\mathrm{t}}^{\mathrm{nr}}}{\Gamma_{\text {bulk }}}=\frac{\Gamma_{\mathrm{b}}^{\mathrm{nr}}}{\Gamma_{\mathrm{bulk}}} .
$$

The experimentally estimated Purcell factor $F_{\mathrm{p}}^{\mathrm{e}}$ may then be obtained from $\Gamma_{\mathrm{t}}^{\mathrm{r}}$ and $\Gamma_{\mathrm{t}}^{\mathrm{nr}}$ by assuming that the background rate is constant and equal to the bulk emission

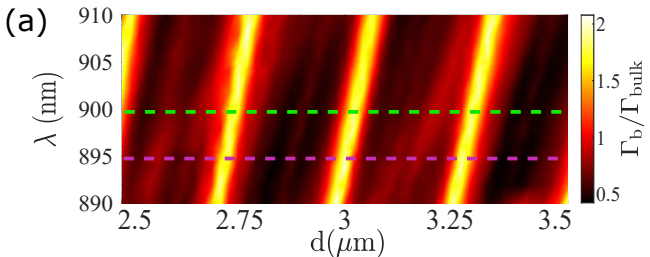

(b)

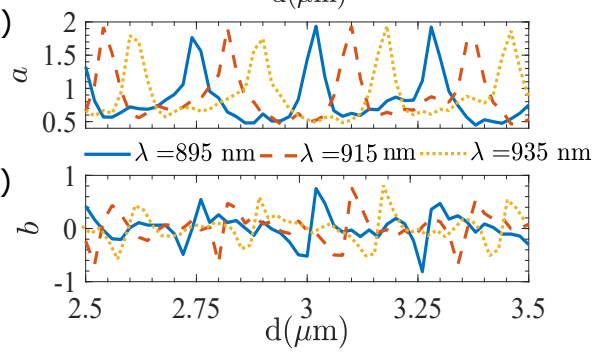

FIG. 4. (a) Normalized background emission rate $\Gamma_{b} / \Gamma_{\text {bulk }}$ versus pillar diameter $d$ and QD emission wavelength $\lambda$ for optimized layer thicknesses with design wavelength $\lambda_{0}=$ $895 \mathrm{~nm}$. (b,c) Correction coefficients $a$ and $b$ defined by Eqs. (8) for $\lambda_{0}=895,915$ and $935 \mathrm{~nm}$ and $\Delta \lambda=\lambda-\lambda_{0}=5$ $\mathrm{nm}$. The resonant (non-resonant) value for $\lambda_{0}=895 \mathrm{~nm}$ is indicated by the purple (green) line in panel (a). $n_{\text {top }}=15$ and $n_{\text {bot }}=2 n_{\text {bot }}$.

rate, such that $\Gamma_{\mathrm{b}}^{\mathrm{r}}=\Gamma_{\mathrm{b}}^{\mathrm{nr}}=\Gamma_{\mathrm{t}}^{\mathrm{nr}}=\Gamma_{\text {bulk }}$. With these assumptions, subtraction of (6) from (5) gives

$$
F_{\mathrm{p}}^{\mathrm{e}}=\frac{\Gamma_{\mathrm{t}}^{r}-\Gamma_{\mathrm{t}}^{n r}}{\Gamma_{\mathrm{t}}^{n r}} .
$$

We now show that $F_{\mathrm{p}}^{\mathrm{e}}$ may differ substantially from the real Purcell factor $F_{\mathrm{p}}$ due to variations in the background emission. The exact Purcell factor $F_{\mathrm{p}}$ can be written in terms of the estimated Purcell factor as $F_{\mathrm{p}}=a F_{\mathrm{p}}^{\mathrm{e}}+b$, where the correction coefficients $a$ and $b$ are determined from the background emission rates as

$$
\begin{aligned}
& a=\Gamma_{\mathrm{b}}^{\mathrm{nr}} / \Gamma_{\text {bulk }} \\
& b=\left(\Gamma_{\mathrm{b}}^{\mathrm{nr}}-\Gamma_{\mathrm{b}}^{\mathrm{r}}\right) / \Gamma_{\mathrm{bulk}},
\end{aligned}
$$

such that the relative error in the estimation of the Purcell factor becomes

$$
\frac{F_{\mathrm{p}}}{F_{\mathrm{p}}^{\mathrm{e}}}=a+\frac{b}{F_{\mathrm{p}}^{\mathrm{e}}} \approx a,
$$

where the last approximation is valid for large Purcell factors.

To examine the influence of the true background emission rate on the relative error, we present in Fig. 4(a) the normalized background emission rate $\Gamma_{\mathrm{b}} / \Gamma_{\text {bulk }}$ as function of physical diameter and QD emission wavelength $\lambda$. As before, the micropillar layer thicknesses are 
diameter-dependent and chosen to place the resonance at $\lambda_{0}=895 \mathrm{~nm}$ (purple line in Fig. 4a) for all diameters. Using temperature tuning, the QD emission may be shifted away from the resonance wavelength, and we now consider a detuning by $\Delta \lambda=\lambda-\lambda_{0}=5 \mathrm{~nm}$ (green line in Fig. 4a). The correction coefficients obtained from evaluation of the background emission $\Gamma_{\mathrm{b}}^{\mathrm{r}}$ on resonance at $\lambda_{0}=895,915$ and $935 \mathrm{~nm}$ and non-resonantly $\Gamma_{\mathrm{b}}^{\mathrm{nr}}$ at $\lambda=\lambda_{0}+\Delta \lambda$ are presented in Figs. $4(\mathrm{~b}, \mathrm{c})$. The correction coefficient $a$ simply equals the normalized nonresonant background rate, and we observe that for a $\Gamma_{\mathrm{b}}^{\mathrm{nr}}$ rate corresponding to a peak in the emission spectrum the true Purcell factor $F_{\mathrm{p}}$ is twice as large as $F_{\mathrm{p}}^{\mathrm{e}}$ estimated using (7). For low Purcell factors, the $b$ correction coefficient should also be taken into account. For $\Gamma_{\mathrm{b}}^{\mathrm{nr}}$ at peak positions, $b$ is always positive leading to an additional increase in the relative error given by Eq. (9). On the other hand, for valleys in the background emission with $a=\Gamma_{\mathrm{b}}^{\mathrm{nr}} / \Gamma_{\text {bulk }} \sim 0.6$, Eq. (9) shows that the Purcel factor may be overestimated by almost a factor of two.

To conclude, we have computed the background spontaneous emission rate into higher-order guided and radiation modes for the micropillar single-photon source. The background rate displays a periodic oscillatory behavio with diameter, where peak positions appear at the onse of new guided modes given by the well-known expression for the cutoff diameter from standard waveguide theory. We thereby provide a guideline for avoiding a potential $\sim 20 \%$ drop in the efficiency for a typical micropillar configuration. We expect that the possibility of optimizing SPS performance through analysis of the background emission rate will be of interest for other SPS designs as well, including as the "bullseye" cavity ${ }^{5,8,9}$ and the tunable open cavity. ${ }^{10}$ In addition, when using the standard experimental procedure for estimating the Purcell factor from experimental lifetime measurements, we have shown how the oscillatory nature of the background emission rate can lead to over- and under-estimation of the Purcell factor by a factor of $\sim 2$. We have presented corresponding correction coefficients relating the estimated value to the true Purcell factor. Finally, a more quantitative understanding of the radiation into the semi-guided mode and of cavity effects on the higher-order guided modes leading to the minor peaks in the $\Gamma_{\mathrm{b}} / \Gamma_{\mathrm{bulk}} \sim 0.6$ valleys is desirable and will be the topic of a follow-up work.

The authors acknowledge support from the China Scholarship Council, from the European Commission via the QuantERA ERA-NET Cofund (HYPER-U-P-S), from the European Research Council (ERC-CoG project UNITY, grant 865230) and from the Independent Research Fund Denmark (grant DFF-9041-00046B).

\section{DATA AVAILABILITY}

The data that support the findings of this study are available within the article.
${ }^{1}$ J. L. O'Brien, A. Furusawa, and J. Vučković, Nat. Photonics 3, 687 (2009), ISSN 17494885

J. W. Pan, Z. B. Chen, C. Y. Lu, H. Weinfurter, A. Zeilinger, and M. Zukowski, Rev. Mod. Phys. 84, 777 (2012), ISSN 00346861 ${ }^{3}$ I. Aharonovich, D. Englund, and M. Toth, Nat. Photonics 10, 631 (2016), ISSN 17494893.

${ }^{4}$ N. Gregersen, P. Kaer, and J. Mørk, IEEE J. Sel. Top. Quantum Electron. 19, 9000516 (2013), ISSN 1077-260X, URL http: //ieeexplore. ieee. org/lpdocs/epic03/wrapper. htm? arnumber $=6493378$ ${ }^{5}$ H. Wang, Y. M. He, T. H. Chung, H. Hu, Y. Yu, S. Chen, X. Ding, M. C. Chen, J. Qin, X. Yang, et al., Nat. Photonics 13, 770 (2019), ISSN 17494893 .

${ }^{6} \mathrm{~N}$. Somaschi, V. Giesz, L. De Santis, J. C. Loredo, M. P. Almeida,

G. Hornecker, S. L. Portalupi, T. Grange, C. Antón, J. Demory, et al., Nat. Photonics 10, 340 (2016), ISSN 1749-4885, URL http://dx.doi.org/10.1038/nphoton.2016.23

X. Ding, Y. He, Z. C. Duan, N. Gregersen, M. C. Chen, S. Unsleber, S. Maier, C. Schneider, M. Kamp, S. Höfling, et al., Phys. Rev. Lett. 116, 020401 (2016), ISSN 10797114.

${ }^{8}$ H. Wang, H. Hu, T. H. Chung, J. Qin, X. Yang, J. P. Li, R. Z. Liu, H. S. Zhong, Y. M. He, X. Ding, et al., Phys. Rev. Lett. 122, 113602 (2019), ISSN 10797114

${ }^{9}$ J. Liu, R. Su, Y. Wei, B. Yao, S. F. C. da Silva Y. Yu, J. Iles-Smith, K. Srinivasan, A. Rastelli, J. Li, et al., Nat. Nanotechnol. 14, 586 (2019), URL http://www nature.com/articles/s41565-019-0435-9.

${ }^{10}$ N. Tomm, A. Javadi, N. O. Antoniadis, D. Najer, M. C. Löbl, A. R. Korsch, R. Schott, S. R. Valentin, A. D. Wieck, A. Ludwig, et al., Nat. Nanotechnol. (2021).

${ }^{11}$ W. L. Barnes, G. Björk, J. M. Gérard, P. Jonsson, J. A. Wasey, P. T. Worthing, and V. Zwiller, Eur. Phys. J. D 18, 197 (2002), ISSN 14346060

${ }^{12}$ J. Claudon, J. Bleuse, N. S. Malik, M. Bazin, P. Jaffrennou, N. Gregersen, C. Sauvan, P. Lalanne, and J. M. Gérard, Nat. Photonics 4, 174 (2010), ISSN 17494885.

${ }^{13}$ J. Bleuse, J. Claudon, M. Creasey, N. S. Malik, J. M. Gérard, I. Maksymov, J. P. Hugonin, and P. Lalanne, Phys. Rev. Lett. 106, 103601 (2011), ISSN 00319007

${ }^{14} \mathrm{M}$. Bayer, T. L. Reinecke, F. Weidner, A. Larionov, A. McDonald, and A. Forchel, Phys. Rev. Lett. 86, 3168 (2001), URL https://link.aps.org/doi/10.1103/PhysRevLett.86.3168.

${ }^{15}$ T. Jakubczyk, H. Franke, T. Smoleski, M. 01ciesiek, W. Pacuski, A. Golnik, R. Schmidt-Grund, M. Grundmann, C. Kruse, D. Hommel, et al., ACS Nano 8, 9970 (2014), pMID: 25181393, https://doi.org/10.1021/nn5017555, URL https://doi.org/10.1021/nn5017555

${ }^{16}$ B.-Y. Wang, E. V. Denning, U. M. Gür, C.-Y. Lu, and N. Gregersen, Phys. Rev. B 102, 125301 (2020), URL https://link.aps.org/doi/10.1103/PhysRevB.102.125301.

${ }^{17}$ U. M. Gür, S. Arslanagić, M. Mattes, and N. Gregersen, Phys. Rev. E (in review).

${ }^{18}$ T. Häyrynen, J. R. de Lasson, and N. Gregersen, J. Opt. Soc. Am. A 33, 1298 (2016), ISSN 1084-7529.

${ }^{19}$ A. Lavrinenko, J. Lægsgaard, N. Gregersen, F. Schmidt, and T. Søndergaard, Numerical Methods in Photonics (CRC Press, 2014), ISBN 9781466563889.

${ }^{20} \mathrm{~L}$. Novotny and B. Hecht, Principles of nano-optics (Cambridge university press, 2012)

${ }^{21}$ J. Liu, M. I. Davanço, L. Sapienza, K. Konthasinghe, J. V. De Miranda Cardoso, J. D. Song, A. Badolato, and K. Srinivasan, Review of Scientific Instruments 88, 023116 (2017).

${ }^{22}$ Y.-M. He, J. Liu, S. Maier, M. Emmerling, S. Gerhardt, M. Davanço, K. Srinivasan, C. Schneider, and S. Höfling, Optica 4, 802 (2017), URL https://www. osapublishing. org/abstract. cfm?URI=optica-4-7-802.

${ }^{23}$ S. Gehrsitz, F. K. Reinhart, C. Gourgon, N. Herres, A. Vonlanthen, and H. Sigg, J. Appl. Phys. 87, 7825 (2000), ISSN 00218979 .

${ }^{24}$ A. Yariv, Optical Electronics in Modern Communications (Oxford University Press, 1997), 5th ed. 
${ }^{25}$ N. Gregersen, S. Reitzenstein, C. Kistner, M. Strauss, C. Schneider, S. Höfling, L. Worschech, A. Forchel, T. R. Nielsen, J. Mørk et al., IEEE J. Quantum Electron. 46, 1470 (2010), ISSN 00189197
${ }^{26}$ G. Lecamp, P. Lalanne, J. P. Hugonin, and J.-M. Gérard, IEEE J. Quantum Electron. 41, 1323 (2005), ISSN 00189197. ${ }^{27}$ C. Böckler, S. Reitzenstein, C. Kistner, R. Debusmann, A. Löffler, T, Kida, S, Höfling, A Forchel, L Grenouillet, J. Claudon, et al., Appl. Phys. Lett. 92, 091107 (2008), ISSN 00036951. 
(a) Infinite nanowire (b) Micropillar
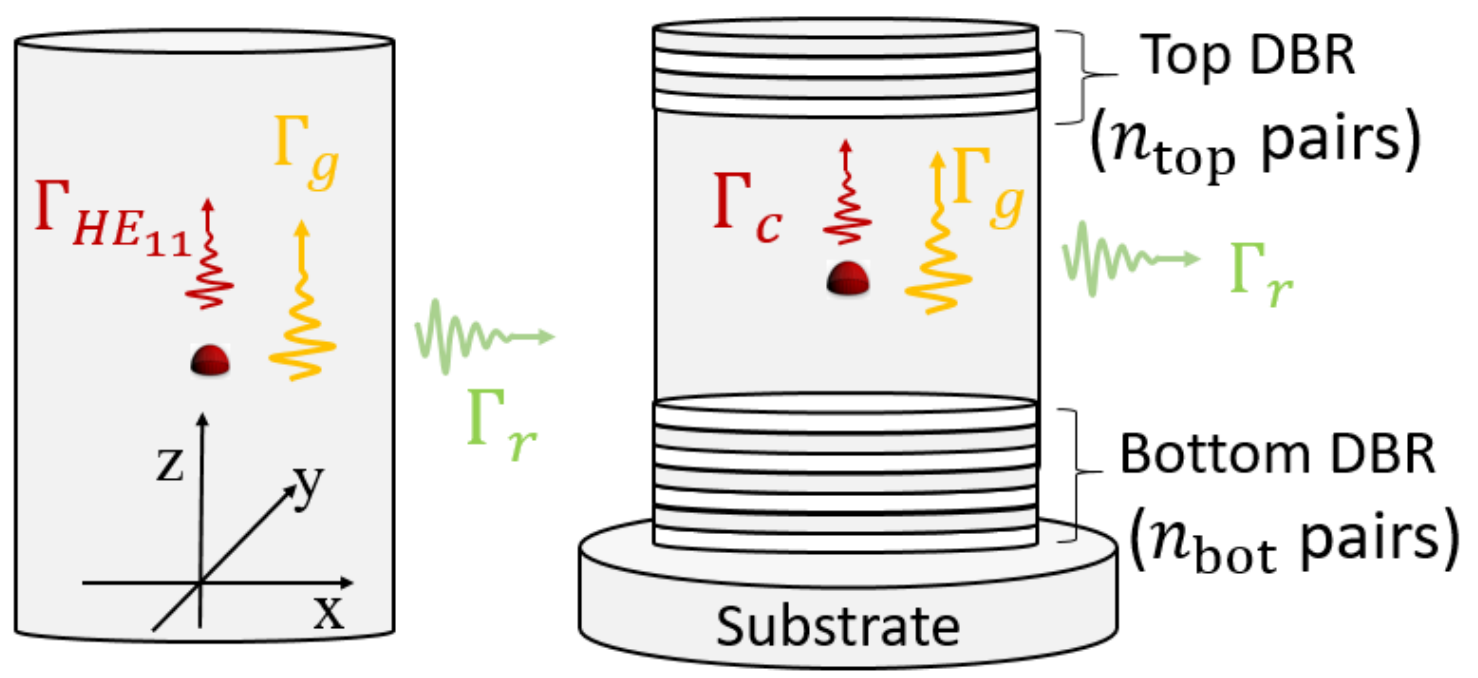


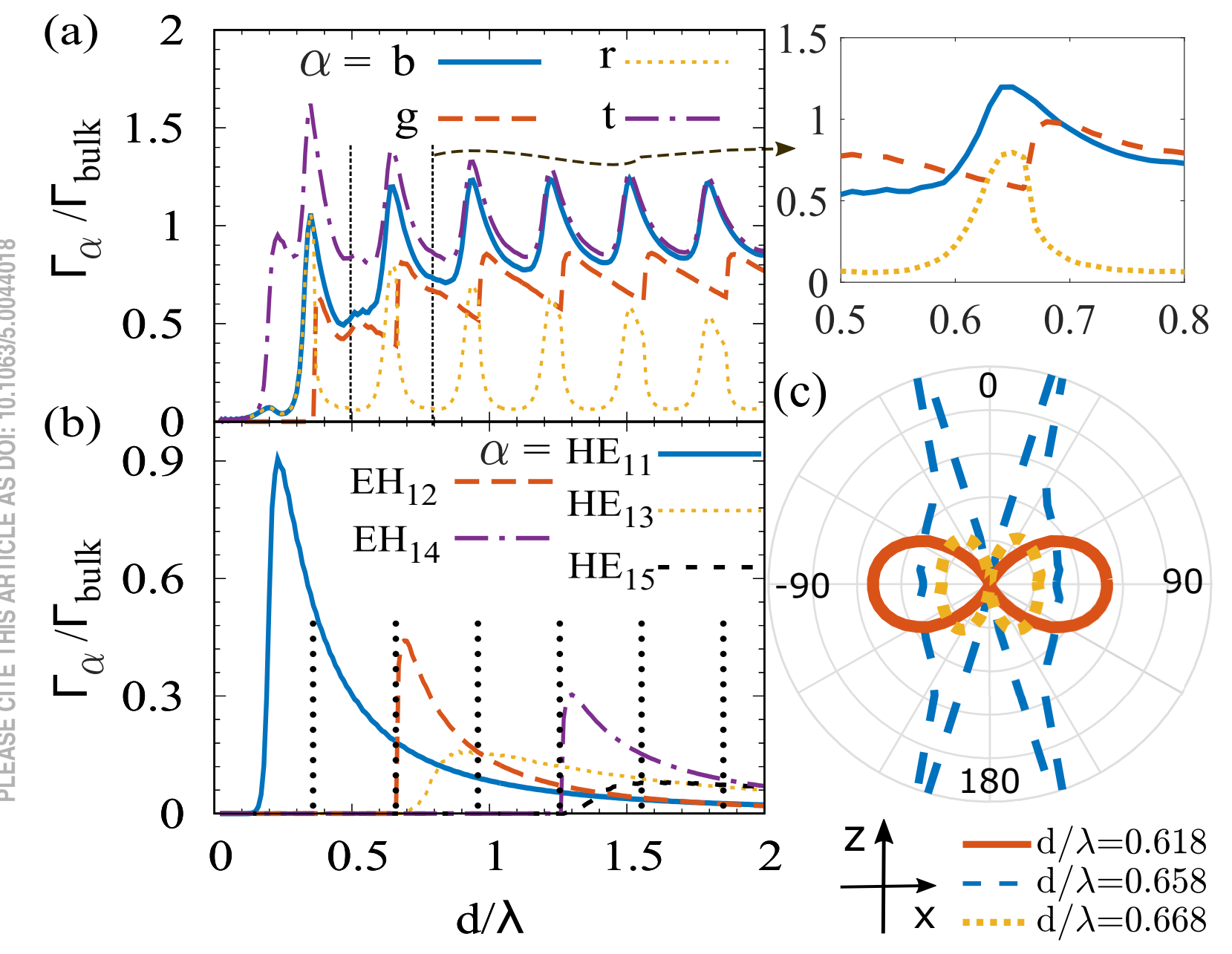




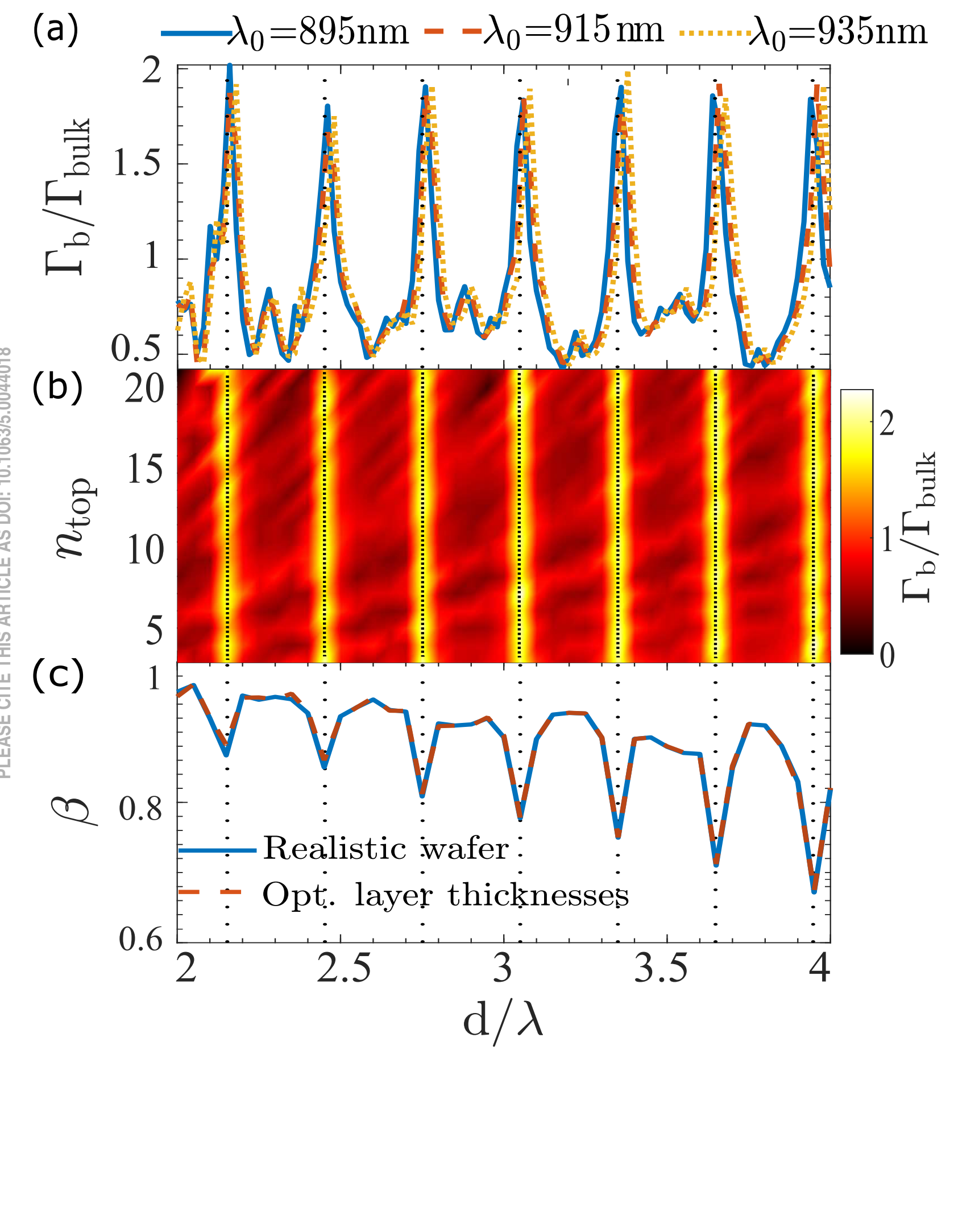



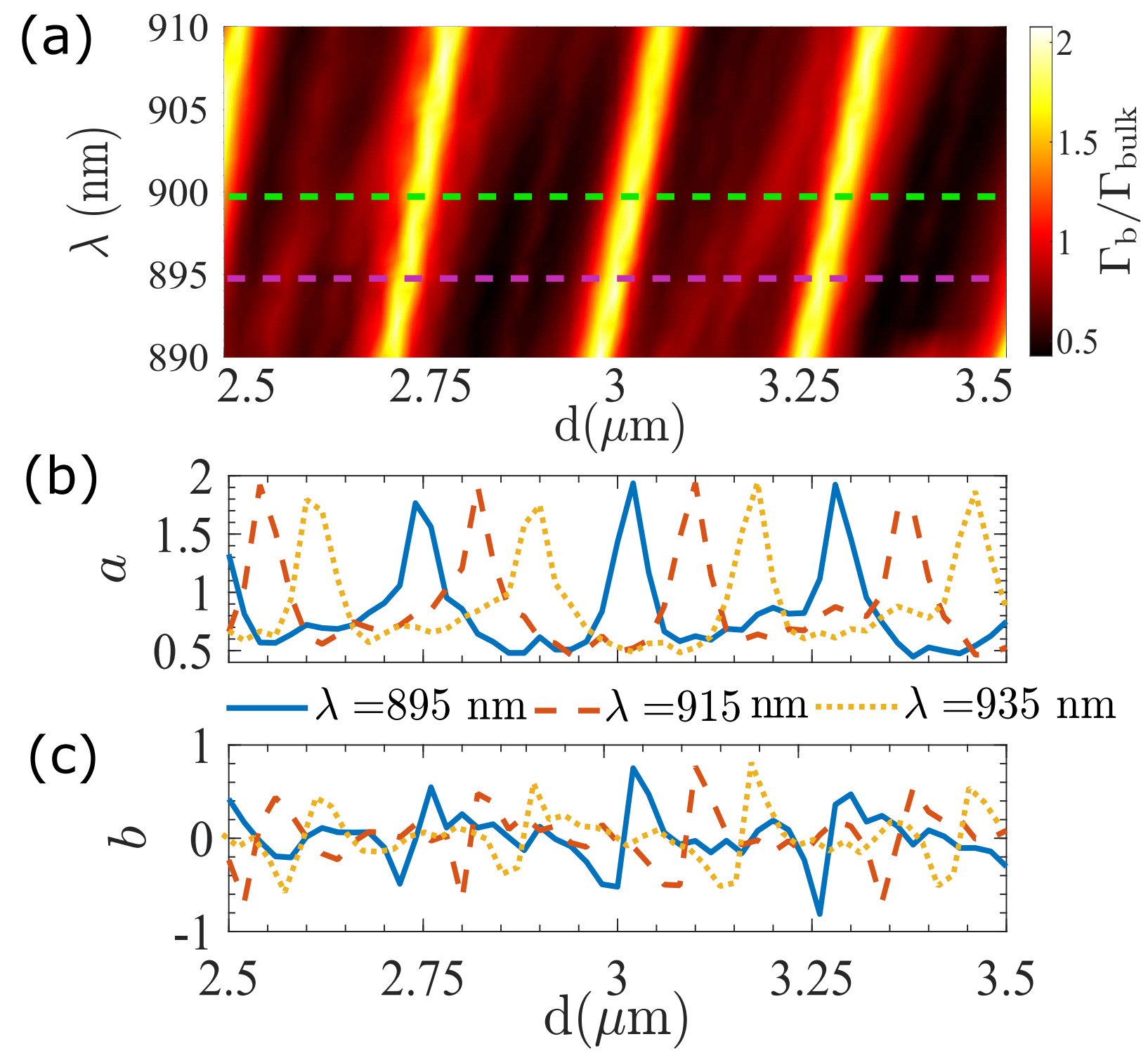